\title{
EL ENFERMO MENTAL. HISTORIA Y CUIDADOS DESDE LA EPOCA MEDIEVAL
}

\author{
$M^{a}$ Elena Pileño Martínez
}

Profesora Asociada de Enfermería Psiquiátrica y de Salud Mental.*

F. Javier Morillo Rodríguez

Profesor Titular de Enfermería Médico-Quirúrgica.*

Paloma Salvadores Fuentes

Catedrática U. en Fundamentos de Enfermería.*

Amparo Nogales Espert

Profesora Titular de Historia de Enfermería.*

* Universidad Rey Juan Carlos, Madrid.

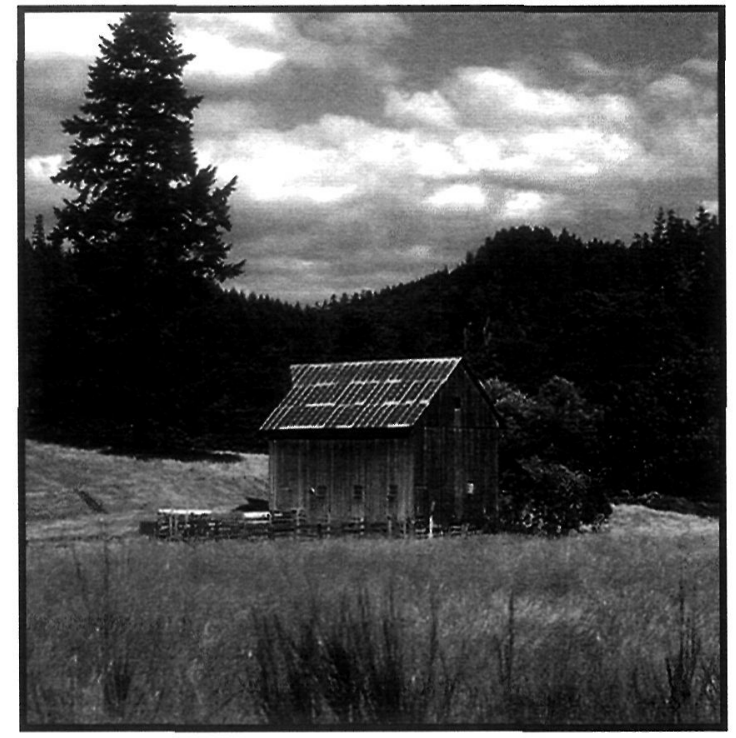

THE MENTALLY ILL. HISTORY AND CARE SINCE MEDIEVAL TIMES

\section{ABSTRACT}

The present work reflects the evolution of insanity in the Spanish cultural, social and historical context. The sequence of events and the interrelation of social systems have improved "assistance", "care" and "medical attention" towards an understanding of the patient's needs and the application of new medical theories.

Nursing role reflects -since the beginning of times- a discipline build by the effort and participation of all those who cared during centuries.
On the other hand, social policies in their integrating role have made insanity adapt to cultural contexts. Throughout the article it is shown how the "insane" have adapted to different forms of institutionalization and disinstitutionalization leading to the current situation.

Key words: History, Insanity, Institutionalization, Confinement, Social.

\section{RESUMEN}

71 presente trabajo plasma la evolución de la locura en España, en un contexto histórico, social y cultural. La sucesión de acontecimientos y la interrelación de los sistemas sociales han hecho que "el trato", " el cuidado" y " la atención médica" caminen hacia una mejora en la comprensión de las necesidades del paciente y en la aplicación de nuevas teorías médicas.

La labor de enfermería, existente desde el principio de los tiempos, refleja una disciplina ganada con el esfuerzo y la participación de todos aquellos que proporcionaron cuidados a través de los siglos.

Por otro lado, la política social en el desempeño de su labor integradora, ha ido adaptando la locura al contexto cultural. En el desarrollo del texto se puede observar cómo "el loco" se ha ido acomodando a las diferentes formas de institucionalización y desinstitucionalización hasta llegar a su situación actual.

Palabras Clave: Historia, locura, institucionalización, confinamiento social. 


\section{INTRODUCCIÓN}

A lo largo de este trabajo queremos mostrar cual ha sido la evolución de la atención al paciente mental.

Hasta hace muy pocas décadas, los recursos asistenciales disponibles para cubrir la asistencia al enfermo mental han sido muy escasos. A esto se le une que la pobreza puede ser un factor determinante en el desarrollo de ciertas patologías, dado que puede implicar aislamiento social, o baja autoestima. Históricamente se puede inferir que las posibilidades de éxito terapéutico se relacionan con las posibilidades económicas o estatus social que el paciente psiquiátrico tenga. El binomio indigente -enfermo psiquiátrico se puede apreciar desde la Edad Media.

Los objetivos de este trabajo son conocer cual es el tratamiento institucional que se le ha dado a la locura desde la Edad Media, así como identificar los recursos disponibles en el tratamiento de estos enfermos y establecer las imágenes sociales sobre " la locura" desde entonces hasta nuestros días.

Partimos de las siguientes hipótesis: La atención al paciente mental se relaciona directamente con el discurso dominante.

El tratamiento del "loco" ha variando desde la Edad Media; desde posturas de reclusión y aislamiento social severo hasta posturas de puertas abiertas.

\section{CUIDADOS EN LA EPOCA MEDIEVAL}

Los hospitales medievales prácticamente nacieron de la caridad cristiana y se podían considerar básicamente de dos tipos: aquellos que estaban situados en las ciudades, siempre fundados por los obispos o custodiados a instancia de los reyes, de la aristocracia del momento o de los municipios; y los situados en zonas rurales, que no eran sino simples dependencias de los monasterios. Estos últimos, dirigidos por religiosos, eran pequeños centros de acogida a enfermos y necesitados, con escasas camas y recursos, donde se llegó contar con personal específico de enfermería y sólo se aportaba consuelo espiritual y cuidados enfermeros, tanto psicológicos como materiales.

El medievo es considerado como época de pobreza, penuria e indigencia. La pobreza podía llevar a la muerte, pero también a la locura, porque solía implicar soledad, rechazo social, sensación de impotencia y pérdida de la autoestima. Los locos, siempre aparecían inmersos en la pobreza, sufriendo tristeza y desesperación. Los pobres se maldecían, blasfemaban y, a veces, perdían la sensatez y caían en la locura.

En 1400, Fray Juan Gilabert Jofré, religioso de la Orden de la merced, y valenciano de nacimiento se inclinó por los cuidados de los enfermos mentales y fue en Valencia, donde convenció a sus habitantes para que se fundase un hospital o una casa que acogiese a los locos de vagaban por las calles de la ciudad, de tal modo que no fuesen sueltos por la ciudad y no pudieran hacer daño.

En 1409 se edificó el hospital de inocentes de Valencia, cuya dedicación exclusiva era la atención de los enfermos locos.

El nombre de Hospitales de Inocentes fue debida a la gran influencia del Cristianismo. Todas las instituciones dedicadas a enfermos mentales se llamaban de inocentes, nombre con que la iglesia designa y evoca el sacrificio de aquellos menores de edad que sufrieron la muerte por el rey Herodes.

En el año 1410 entró en funcionamiento el Hospital de Santa María o de Inocentes, el primer manicomio del mundo cristiano-occidental, con el propósito de resolver una necesidad social. Todo dependía de las ordenes dadas por el hospitaler. A los internos, se les obligaba a trabajar en la granja, en el jardín, en la limpieza y en los servicios, si eran hombres; cosiendo, tejiendo o confeccionando vestidos, si eran mujeres. Se trataba siempre de evitar la inactividad, pues, según se creía, la permanente ociosidad podía perturbarlos más, fomentándoles el vicio y los malos hábitos.

Si eran desobedientes o se mostraban furiosos, se les azotaba, se les colocaban grilletes, incluso de forma permanente o se les encerraba en jaulas o gavias. Se les vestía uniformadamente con sayos hechos de jirones y con una caperucilla de loco. Esos sayos de tela gruesa eran sustituidos por otros de colores más vistosos en los días de fiesta.

La fundación del primer manicomio en Valencia supuso la cristalización de una nueva concepción social de la locura. Desde finales del siglo XIII la Ciudad de Valencia se había convertido en un importante centro comercial, siendo distribuidora de productos a Castilla, a Italia, y al 
norte de Africa, reemplazando a Barcelona en su posición hegemónica.

También fue el punto de mira para la creación de otras instituciones parecidas ; en 1436 se fundó otro manicomio en Sevilla, ciudad que desde el año 1356 pertenecía a la Corona de Castilla y que, por su proximidad a Portugal, Norte de Africa y Canarias, se había convertido en un populoso centro de actividad comercial.

En 1483, durante el reinado de los Reyes Católicos, se fundó en Toledo otro Hospital de Inocentes y locos, promovido por el Nuncio y canónigo de la Catedral don Francisco Ortiz, para lo que cedió varias casa de su propiedad. Por tanto, el nuevo hospital dependía de la Iglesia.

En 1489 se fundó en Valladolid otra casa de locos, también administrado por la Iglesia y concretamente por el cabildo catedralicio de Valladolid.

Durante el siglo XV continuaron proliferando por doquier los hospitales urbanos, hasta tal punto, que se llegó a la necesidad de integrar los pequeños hospitales y convertirlos en hospitales generales, con secciones o departamentos para dementes y locos.

Mas tarde, se inicia la concentración hospitalaria. Barcelona, pionera en el intento absorbió 6 hospitales, creándose el Hospital de la Santa Creu, que ya desde su comienzo, pagaba a dos médicos, y contaba con dos departamentos o secciones para el asilo de dementes, además de otras salas para enfermedades internas, mujeres embarazadas, leprosos, sifilíticos, ...

En 1425, el Rey aragonés Alfonso V el Magnánimo fundó en Zaragoza el Hospital de Nuestra señora de Gracia, un moderno hospital general que admitía toda clase de enfermos sin distinción de sexo o creencia. Incluía una casa de locos edificada a comienzos del siglo XV por la iniciativa de un grupo de ciudadanos, y luego ubicada en el marco del hospital general.

El Gran Consell General de Baleares, unificó todos los hospitales de la isla, en uno sólo. Contaba con dos salas; una para dementes del sexo masculino, de aspecto agradable y bien distribuido y otra para las de mujeres, donde habitualmente los exorcistas conjuraban a las enfermas.

\section{LA EDAD MODERNA}

El reinado de los Reyes Católicos, del año 1474 al 1505 , supuso el fin de la reconquista, la unificación de los reinos hispánicos, la consolidación del poder real, la consecución de la unidad religiosa, la expulsión de los judíos, la persecución de los conversos, la Inquisición y el inicio de la cultura renacentista... Bajo su mandato, la edad moderna, acentúa el proceso de concentración hospitalaria, sobre todo en las grandes ciudades, y una progresiva secularización.

España situada ya en la edad moderna y con tres instituciones específicas para el tratamiento de locos en inocentes (Sevilla, Toledo y Valladolid) y siete hospitales generales con departamentos para dementes (Barcelona, Zaragoza, Valencia, Palma de Mallorca, Lérida, Granada, y Córdoba). Poseía la situación mas avanzada de Europa.

En 1526, Luis Vives, pensador católico, propuso registrar a todos los pobres que mendigaban por las calles, los que sufrían sus necesidades en sus casas, o los que vivían en hospitales. No se debía permitir estar ocioso a ningún pobre que estuviera capacitado para trabajar. Sólo los mendigos suficientemente enfermos deberían ocuparse de trabajos mas o menos livianos, según su edad y estado de salud. En cuanto a los pobres privados del uso de razón, habían de ser tratados con sumo cuidado y con todo respeto

La locura debía de hallar acogimiento en el hospital, había que excluir al loco, de entre los pobres; era necesario desarrollar una medida de saneamiento que lo pusiera fuera de la circulación social.

Durante la edad moderna, la imagen de la locura esta mas presente en el paisaje cultural de la época, el tratamiento de las enfermedades seguía sin ser una actividad exclusiva de los médicos con titulación universitaria y de los prácticos reconocidos por las autoridades. Aparecían los sanadores o curanderos, que gozaban de un gran reconocimiento social sobre todo fuera de las ciudades. La escasez de médicos y la ineficacia de la medicina unido a la superstición de la gente hacía aceptar con facilidad lo supuestamente divino, diabólico y lo mágico de vida cotidiana.

Durante el siglo XVII la atención de enfermería se basaba en el cuidado de los enfermos. La ali- 
mentación en la casa era sana, abundante y variada, y se ponía especial esmero en la limpieza corporal de los pacientes mediante el baño, apartando a los que en las crisis estaban furiosos y sucios .

El personal estaba compuesto por los siguientes funcionarios: Administrador o Mayordomo, procurador, escriba, hospitaler, y dependientes. Las funciones de cuidado las realizaban el hospitaler y el dependiente atendiendo las necesidades básicas de los enfermos. Los dependientes aparecen en los hospitales medievales valencianos como encargados de las necesidades básicas de los enfermos; higiene, alimentación, eliminación, movilización y su cuidado permanente, es decir, realizando funciones de enfermería.

Durante el siglo XVIII, aumentó la población española, desaparecieron las hambrunas y las grandes epidemias del pasado, creció la producción económica y surgieron expectativas de desarrollo de los recursos existentes.

En este siglo, los hospitales continuaban, impregnados de caridad religiosa, pero a lo largo del siglo fueron cada vez mas controlados por los poderes públicos.

En 1766 se creó el Hospital General de Madrid con muy escasas rentas, disponía de 38 salas con un total de 1.564 camas, de las cuales 38 eran para locos, canalizando el grueso de la asistencia hospitalaria de Madrid, pero el mal estado sanitario y la inexistencia de cuidados enfermeros y médicos convertían las enfermedades mas simples en graves y agudas, entre otras cosas, por el aire excesivamente contagiado.

En Sevilla, a mediados del mismo siglo, los locos rara vez salían del Hospital de Inocentes. Se rompió la tradición secular por la que la mayoría de los locos salían a pedir limosnas en las calles de la ciudad. El confinamiento aumentaba y los espacios para ellos iban escaseando.

Los enfermos quedaban drásticamente aislados del exterior, gobernados por la autoridad del administrador, éste, residía en el hospital y ganaba un salario por su trabajo, ejerciendo un control estricto de los enfermos. Siguiendo sus ordenes los enfermeros debían cuidar y vigilar en todo momento a los internos en sus habitaciones.

\section{EDAD CONTEMPORÁNEA}

En el último tercio del siglo XVIII, hubo cambios administrativos y policiales introducidos por Carlos III, el ingreso de los dementes debía de hacerse a instancias de los alcaldes de barrio y otras autoridades civiles, que sobre todo debían tener en cuenta el peligro de hacer violencia en otras personas o en las propiedades de otros.

En Valencia también era preciso el certificado médico para el ingreso y para la salida de los dementes del Hospital General de Valencia y lo mismo sucedía en el de Inocentes de Sevilla. Sin embargo, los médicos casi no tenían mayor intervención administrativa en los citados establecimientos.

Los médicos higienistas manifestaban que el desorden social y moral producía, alteraciones importantes en la conducta de los obreros. La locura se presentada estrechamente ligada a alteraciones morales generadas por factores sexuales, formas de vida desintegradas, problemas económicos, relaciones familiares alteradas. Se hablaba mas de prevenir que de curar, y proponían como remedio mas eficaz el mejorar las condiciones higiénicas de la población, especialmente del proletariado industrial.

Los alienistas franceses, y por su influencia, también los españoles, estaban de acuerdo con los higienistas en cuanto a la importancia que daban a las condiciones sociales y a las causas morales de la producción de la locura, y en la necesidad de tratar a los enfermos mentales, moralizándolos, tutelándolos, confinándolos.

En el siglo XIX, la Ley de Beneficencia ,establecida en 1836 por el gobierno progresista de Mendizábal, ordenaba la existencia de los hospitales públicos, entre los que debían diferenciarse los establecimientos especiales para el tratamiento de los locos.

En la mitad del XIX, España, toma conciencia del número de ingresos de enfermos mentales. Éste había crecido de forma considerable en las diversas instituciones de reclusión, haciéndose aún mas precaria la situación.

España, bajo la influencia francesa imitaba las reformas que ésta realizó en la primera mitad de siglo, consideró la locura como una amenaza social y definió un espacio específico de confinamiento 
para ella, aunque se tratara de darle un carácter médico. Se trataba de proteger a la sociedad del loco y a éste de la enfermedad que padecía, en un espacio en el que la locura era transitoria y curable.

Posteriormente, Philippe Pinel, médico de Bicètre, iniciador del alienismo, como rama autóctona de la medicina, reflejó la necesidad de objetivar científicamente la enfermedad y la integración administrativa de la locura hasta entonces marginada. Pinel dividía la enfermedad de la locura en cinco formas: la melancolía, la manía sin delirio, la manía con delirio la demencia, y el idiotismo.

El principal remedio para la locura sólo podía hallarse en un hospital bien organizado, y consistía en dominar y domar al loco. Un hospital de locos, además de estar bien situado y disponer de un recinto vasto y espacioso, debía ser construido de manera que permitiera la separación de las diversas clases de locos, evitando que éstos se relacionasen entre sí, con el fin de prevenir la recaída.

En nuestro país, el estado se mostraba incapaz de mantener el orden público. El desinterés por la creación de nuevos manicomios desembocó en la posibilidad de creación de manicomios o clínicas privadas con la pretensión de ofrecer practicas asistenciales modernas y trato humanizado a los enfermos. El primer centro se creó en 1844 en Lloret de Mar, fundado y dirigido por el médico Francesc Campdera.

El manicomio se dividía en dos grandes departamentos, una para hombres y otra para mujeres. Funcionaba, o pretendía funcionar, según el sistema del "non restraint "del inglés Connally. Lo importante para la organización terapéutica era la salud corporal, la alimentación y la educación tanto oral como física.

Se busca sobre todo la tranquilización del enfermo, no había métodos correctivos, únicamente con paciencia, bondad, actuaciones cuidadosas, limpieza y buen sentido se cuidaba al enfermo. Había habitaciones acolchadas para casos de agitación. Allí el paciente vivía en un nuevo sistema moral, se decía que el servicio facultativo lo componía el médico propietario, un médico residente, un boticario, un practicante, y los correspondientes enfermeros y enfermeras. Además existía un capellán, encargado de enseñar la doctrina cristiana a los señores pensionistas.
Con esta oferta asistencial aumentó el número de ingresos. En 1859 se logró un convenio con el Gobierno para el ingreso de enfermos judiciales a cargo del estado. A partir de ahí la situación comenzó a deteriorarse.

En 1879 se hizo una estadística de dementes a escala nacional, y se encontró que, desde 1860, la población reclusa se había triplicado. El gobierno instaba para que las diputaciones construyesen nuevos manicomios. El aumento de los ingresos incrementaba los costes de las instalaciones, y no había recursos suficientes para sufragar los gastos para su ampliación y mejora.

El modelo del manicomio moral, importado de Francia, no se pudo implantar en España, pese a los esfuerzos teórico - prácticos de algunos alienistas, ya que el número y la calidad de los manicomios no se correspondía con las necesidades de la población, sino con el grado de civilización alcanzado. Por esto, en España había pocos manicomios y de escasa calidad.

En 1914 se empieza a desarrollar la enfermería mental de carácter laico. Se sigue custodiando a los locos en dos sistemas: el penitenciario y el sanitario.

En 1924 se crea la Escuela Nacional de Sanidad y en 1926 se inaugura una escuela de Psiquiatría dotada con toda clase de elementos para la investigación científica del enfermo mental y su tratamiento y curación. A partir de este momento sería requisito indispensable la posesión de estos estudios para poder acceder legalmente al cuidado de los enfermos mentales. La profesionalización de la enfermería psiquiátrica supuso un avance en la historia de la enfermería en España.

En julio de 1931 el Gobierno Provisional promulgó un nuevo decreto de internamiento de enfermos psíquicos, que recogía casi íntegramente el anteproyecto de la Liga; "todo enfermo psíquico debía recibir asistencia médica en España, bien en el medio familiar, bien en un establecimiento público o privado, cuya organización técnica correspondería al estado actual de la ciencia psiquiátrica".

En casos especiales, las clínicas y hospitales podían funcionar en las ciudades, con un carácter exclusivamente abierto, sujetos al reglamento general de la asistencia hospitalaria, mientras que 
los establecimientos alejados de los centros urbanos podrían conservar su carácter cerrado o asilar.

Se preveían tres supuestos de admisión de los enfermos; por voluntad propia, por indicación médica o por orden judicial o gubernativa.

El primer paso para la reforma psiquiátrica fue la puesta en marcha de una estadística nacional: en 1933 la dirección general de Sanidad contabilizó un total de 24.554 enfermos acogidos en los establecimientos psiquiátricos españoles

En 1932 se creaba por decreto el primer Dispensario de Higiene mental de Madrid, como centro piloto. Su misión específica consistía en el estudio de los procesos iniciales, el tratamiento ambulatorio de los casos leves y las curas de reposo en servicio abierto de los enfermos neuróticos pobres.

En conclusión se pretendió mejorar los manicomios descongestionándolos y convirtiéndolos en hospitales psiquiátricos activos. Sin embargo, en cinco años los resultados prácticos de la reforma psiquiátrica, eran muy pocos.

Los presupuestos eran escasos y esto chocaba con el incremento en la demanda de internamiento. El hacinamiento de los enfermos en los manicomios hacía inviable la práctica de una terapéutica eficaz, tal como ocurrirá en Valencia, cuyo manicomio albergaba en 1936 a 1184 enfermos.

En 1936, la guerra civil española fue una inmensa locura colectiva, por la que muchísima gente hubo de sobrevivir en circunstancias extraordinariamente tensas y precarias, al límite de sus posibilidades y recursos psicológicos.

No había datos precisos y homologables para evaluar la frecuencia de enfermedades mentales habidas en la Guerra Civil, y la interpretación de los pocos que existían fue, luego, políticamente muy sesgada; sin embargo, los psiquiatras de la época, afirmaron luego que no había habido un aumento significativo de las auténticas enfermedades mentales, en todo caso la guerra sólo había actuado como reveladora de enfermedades.

En noviembre de 1936 Madrid se quedó con la clínica Psiquiátrica del Hospital Provincial como único centro de internamiento psiquiátrico. La precariedad en los tratamientos afectaba también a los enfermos, ya que sólo se les aplicaba las tradicionales medidas de contención. A partir de 1940 eran introducidas la insulina, el electroshock y las lobotomías como tratamiento para los pacientes con larga hospitalización.

El personal de los hospitales iba disminuyendo dramáticamente, y estos tratamientos, en general en toda Europa, fueron fundados con muy poca consideración en lo que se refiere a los cuidados. Por ejemplo, las enfermeras debían de participar en estos tratamientos y responsabilizarse de ellos de forma natural, como una función suya más.

En 1943 la dirección General de Sanidad decretó la creación de dispensarios de higiene mental, en todas las jefaturas Provinciales de Sanidad, pero hasta 1950 no se proveyeron las correspondientes plazas de personal sanitario, y sólo en la mitad de las provincias españolas.

En 1950 entra el primer antipsicótico, la clorpromazina, introducido por el laboratorio Smith-kline and French. Este mismo laboratorio también financió la película "La relación enfermera-paciente" cuyo trabajo fue dirigido por Hildegard Peplau, Directora de la Asociación americana de enfermeras en 1969 , enfermera doctora aventajada que dirigió su objetivo hacia diferentes facetas en la formación y cuidado de las enfermeras psiquiátricas.

España había partido con mucho retraso respeto a otros países. Se crea, en 1970, la especialidad de Ayudante Técnico Sanitario Psiquiátrico con dos cursos de ocho meses de duración.

En 1985, el Ministerio de Sanidad, elaboró un documento para la Reforma Psiquiátrica y la atención en la Salud Mental que publicaba la plena integración de la Salud Mental en la asistencia sanitaria general. Las comunidades autónomas siguieron la pauta indicada, aunque existió desigualdad entre ellas. Unas comunidades tuvieron una amplia gama de recursos mientras que otras poseían una elevada carencia.

\section{LA REFORMA Y LAS COMUNIDADES TERAPÉÚTICAS}

Han pasado mas de 4 décadas desde que comenzó el proceso de desinstitucionalización del enfermo mental. Se cambiaron los cuidados y el soporte de la custodia psiquiátrica por las diferentes comunidades. Después de un tratamiento prolongado, el efecto que ha tenido para los pacien- 
tes ha sido diferente dependiendo de cada país.

A nivel mundial, el proceso de desinstitucionalización es parecido. Los cuidados de salud mental dependen de factores como la aceptación social y del derecho de los enfermos mentales a ser miembros de la comunidad.

En muchos países todavía tienen los cuidados de psiquiatría habituales; un 38\% de países del mundo todavía no han instaurado los servicios de salud mental comunitarios. Otros países todavía no lo tienen instaurado aunque se comprometieron a dicha cobertura, y solamente lo mantienen en áreas urbanas (Argentina, India, Nigeria, Rusia, Turquía).

En Europa, la situación varía dependiendo de los países. En Inglaterra, por ejemplo, al igual que USA, las camas han ido disminuyendo, las comunidades mentales han sido establecidas por todo el país. El gobierno adoptó cuidados prácticos para tratar a los enfermos mentales orientados a la seguridad y el conservadurismo.

La desinstitucionalización avanza de diferentes formas dependiendo del nivel de recursos. USA piensa que la coordinación de los recursos y la incorporación de empresas u organismos públicas y privadas es necesaria para la desinstitucionalización de los enfermos mentales. Otros han notado que la desinstitucionalización ha puesto mucho hincapié en el cuidado de los locos y no en las pequeñas atenciones humanas que serían mas efectivas y darían mas calidad al cuidado.

\section{BIBLIOGRAFÍA}

- Charles Marie, F. Ed. Prensa Médica Mexicana S.A.(1987). " Desarrollo histórico de la enfermería".

- De la Vega, J. , Esteban, A. (2001). " Historia de la enfermería en Castilla y León: practicantes y enfermeras". Revista - Centro de Salud- Noviembre

- Donahue,P. Ed. Doyma.1993. Historia de la Enfermería.

- Fakhoury, W; Priebe, E. (2002 )“ The process of deinstitutionalization: an international overview”. Current opinion in Psychiatry,. 15 (2) March,. Pp 187-192.

- Ferrer Caro, F. (2001) “El cuidado en la España Medieval". Revista Index de enfermería. (34). Otoño.

- García Hernández, F; Pancorbo Hidalgo, P; Rodríguez Torres, C. (2001). "Evolución de la enfermería profesional en España". Revista Index de Enfermería.( 32-33) primavera-verano

- González Duro, E. Ed. Temas de Hoy . (1994.) “ Historia de la locura en España”. Tomo I,II.III. Madrid
- González Duro, E. ( 2000). “ De la psiquiatría a la Salud Mental" . Revista de la asociación Española de Neuropsiquiatría". Junio

- Hernández Conesa, J. Ed. Interamericana McGraw -Hill (1999). "Historia de la enfermería: un análisis histórico de los cuidados de enfermería". Madrid .

- Huertas, R. Ed: Fondo de investigaciones Sanitarias de la Seguridad Social. 1992. "Del manicomio a la Salud Mental". Madrid

- Iradj, M.; Herzl, M; Guido, Ph:D. (1973). “ The social Acceptance of the Ex -Mental Hospital Patient. Community Mental Health Journal. (9).

- Nogales, A. (1 ${ }^{\circ}$ semestre 2001 ) "La Enfermería y el cuidado de los enfermos mentales en el Siglo XV". Revista -Cultura de los cuidados. Revista de humanidades- (9).

- Shirley A. Smoyayak, RN,PhD, Faan. "The History, Economics, and Financing of Mental Healt Care". Part 3. Journal of Phychosocial Nursing, 38(11). Pp 33-41.

- Siles. J. Ed. Aguaclara (1999). " Historia de la Enfermería".Alicante

- Siles, J ; Cibanal, L; Vizcaya, F; Solano, C ; García, E; Gabaldón E (2001). " "De la custodia a los cuidados : una perspectiva histórica de la enfermería en Salud Mental". Revista -Cultura de los cuidados. Revista de humanidades y enfermería- (9). $1^{\circ}$ Semestre

- Solana, I. Rodríguez, I.García, J.; (2001). "Evaluación de la aplicación de principios básicos normados por al O.M.S. para la atención de la Salud Mental”. Revista -Cuadernos de Bioética- Madrid

- Ventosa Esquinaldo, F. (1997) "El enfermo mental en el siglo XV en España. Conceptuación como enfermo distinto y curable. Su cuidado." Revista: Cultura de los cuidados. Revista de enfermería y humanidades. Pp. 43-61.

- Ventosa Esquinaldo, F. Ed. Ciencia 3.(1984.) “ Historia de la enfermería española"-. Madrid. 